\title{
A Test Statistic for Weighted Runs
}

\author{
Frederik Beaujean*, Allen Caldwell \\ Max Planck Institute for Physics
}

\begin{abstract}
A new test statistic based on runs of weighted deviations is introduced. Its use for observations sampled from independent normal distributions is worked out in detail. It supplements the classic $\chi^{2}$ test which ignores the ordering of observations and provides additional sensitivity to local deviations from expectations. The exact distribution of the statistic in the nonparametric case is derived and an algorithm to compute $p$-values is presented. The computational complexity of the algorithm is derived employing a novel identity for integer partitions.
\end{abstract}

Keywords: Success runs, $p$-value, $\chi^{2}$, Integer partitions, Measurements with Gaussian uncertainty

2000 MSC: 62G10, 05A17, 60C05, 62P35

\section{Introduction}

In the course of scientific inference, we are faced with one basic task: comparing observations and model predictions. Based on this comparison, the hypothesized model may be either accepted or rejected. In the latter case usually an improved model is sought. The comparison between observations and the new model is then repeated until a satisfactory model has been constructed.

In model validation the goal is to provide quantitative test procedures. The standard approach consists of defining a scalar function of the data $D$, called test statistic $T(D)$, such that a large value of $T$ indicates a large

\footnotetext{
${ }^{*}$ Corresponding author

Email addresses: beaujean@mpp.mpg.de (Frederik Beaujean), caldwell@mpp.mpg.de (Allen Caldwell)
} 
deviation of the data from the expectations under the hypothesized model $\mathcal{H}$. Correspondingly, small $T$ is seen as good agreement. Let $T_{o b s}$ denote the value of $T$ observed in the actual data set. In order to facilitate the interpretation of $T$ (how large is too large?), it is useful to introduce the $p$-value. Assuming $\mathcal{H}$, the $p$-value is defined as the tail area probability to randomly sample a value of $T$ larger than or equal to $T_{o b s}$ :

$$
p \equiv P\left(T \geq T_{o b s} \mid \mathcal{H}\right)
$$

If $\mathcal{H}$ is correct and all parameters are fixed, then $p$ is a random variable with uniform distribution on $[0,1]$. An incorrect model will typically yield smaller values of $p$. This is used to guide model selection. For the same data, different models will give different $p$. Similarly, a different choice of the test statistic produces a different $p$ for the same model and data. Why use different statistics? Because one statistic is sensitive to certain, but not to all properties of the model.

To illustrate this, recall that in the majority of practical applications the hypothesis $\mathcal{H}$ describing the set of $N$ observations $D=\left\{X_{i}\right\}$ is constructed with individual observations $X_{i} \in \mathbb{R}$ considered independent. The discrete scalar index $i$ provides an ordering for the data. It may represent time, length, energy .... For concreteness, let us assume independent, normally distributed variables $X_{i} \sim \mathcal{N}\left(\mu_{i}, \sigma_{i}^{2}\right)$. We can write the probability density of the data as

$$
P(D \mid \mathcal{H})=\prod_{i=1}^{N} P\left(X_{i} \mid \mu_{i}, \sigma_{i}^{2}\right) \propto \prod_{i=1}^{N} \exp \left(-\frac{\left(X_{i}-\mu_{i}\right)^{2}}{2 \sigma_{i}^{2}}\right)=\exp \left(-\frac{\chi_{N}^{2}}{2}\right)
$$

where $\chi_{N}^{2}=\sum_{i=1}^{N} \frac{\left(X_{i}-\mu_{i}\right)^{2}}{\sigma_{i}^{2}}$ appears naturally; it is the most widely used test statistic to probe $\mathcal{H}$; a large $\chi_{N}^{2}$ translates directly into a small $P(D \mid \mathcal{H})$. Note that $\chi_{N}^{2} / N$ is a measure of the average deviation per observation, but it is blind to the ordering of the data points.

In this paper, we introduce a test statistic sensitive to local deviations of the data from expectations within an ordered data set. The test statistic we propose is valid for data which are expected to have equal probabilities to be below or above expectations. For concreteness, we consider the $X_{i}$ normally distributed with known mean and variance, but the formulation is valid for any symmetric distribution. 
Statistics involving runs; i.e. sequences of observations that share a common attribute commonly called a success, have drawn a lot of attention. Good reviews are presented in [1, 2, 3, 4]. Most of the early work was centered around independent Bernoulli trials; cf. [5, 6] and [7]. After the introduction of the Markov chain imbedding approach by [8], runs statistics have been considered also for more complicated models with Markov dependence [9, 10, 11, 12]. For the case of exchangeable binary trials see [13]. Ref. 14 provides a summary of useful formulae and distributions using a combinatorial approach.

In this paper we call an observation a success, $\mathrm{S}$, if the observed value exceeds the expected value. Similarly an expected value exceeding the observation is considered a failure, $\mathrm{F}$. Obviously the meaning of success and failure may be reversed, and without loss of generality we may concentrate on the success runs. Using the notation of [4] and counting convention of [1], the simplest test statistics based on runs are the number of runs of length exactly $k, E_{N, k}$, and the length of the longest run, $L_{N}$. As an example consider the realization FSSFS; then $E_{5,1}=1$ and $L_{5}=2$. Observe that both $E_{N, k}$ and $L_{N}$ ignore relevant information: a success is a success no matter how much $X_{i}$ is bigger than its expected value.

The goal of this paper is to enhance the existing procedures based on $E_{N, k}$ or $L_{N}$ by introducing a new runs statistic $T$, similar in spirit to $L_{N}$, which includes that extra information. For simplicity, we construct the statistic only for success runs; the same steps can be taken to define an analogous statistic for failure runs as well. $T$ is formally defined in three steps:

1. Split the data $\left\{X_{i}\right\}$ into runs. Keep the success runs and ignore the failure runs. Denote by $A_{j}=\left\{X_{j_{1}}, X_{j_{2}} \ldots\right\}$ the set of observations in the $j$-th success run.

2. Associate a weight with each success run. The weight $w\left(A_{j}\right)$ ought to be chosen such that a large weight indicates large discrepancy between model and observations. A natural choice of the weight function is a convenient one-to-one function of the probability (density) of $A_{j}$ such as $w\left(A_{j}\right)=\left[P\left(A_{j} \mid \mathcal{H}\right)\right]^{-1}$ or $w\left(A_{j}\right)=-2 \log \left(P\left(A_{j} \mid \mathcal{H}\right)\right)$.

3. Choose $T$ as the largest weight:

$$
T \equiv \max _{j} w\left(A_{j}\right) .
$$

We proceed as follows. In sec. 2 we first derive the general expression for $p=P\left(T \geq T_{o b s} \mid \mathcal{H}\right)$ given a model with independent observations and 
equal probability of success and failure. The formulation is true for arbitrary weights. Next we give explicit results in one concrete example of great importance where $X_{i} \sim \mathcal{N}\left(\mu_{i}, \sigma_{i}^{2}\right)$ with $\mu_{i}, \sigma_{i}^{2}$ known and $w\left(A_{j}\right)$ chosen as the sum of $\chi^{2}$ 's of the samples in $A_{j}$. For a large number of observations, $N \gtrsim 80$, the evaluation of the exact expressions for $p$ turns out to be highly demanding both in terms of computer time and memory, as it scales with the number of integer partitions. Thus we present a Monte Carlo method that works even for $N \gtrsim 1000$ and compare exact and approximate results. A selection of critical values of $T$ for common confidence levels is tabulated. The power of $T$ is studied in sec. 3. Compared to $\chi^{2}$, tests based on $T$ are superior in detecting departures from $\mathcal{H}$. This is demonstrated with a specific but commonly arising example - the presence of an unexpected localized peak. As final remarks, we discuss generalizations of $T$ to non-symmetric uncertainties and composite hypotheses (parameters fit) in sec. 4. In the appendix we introduce integer partitions in more detail and derive the recurrence relation for integer partitions needed to analyze the computational complexity required for computing $p$-values for $T$.

\section{Runs statistic}

Let us now make the definition of $T$ explicit in the following example. The hypothesis $\mathcal{H}$ for the data $\left\{X_{i}\right\}, i=1 \ldots N$ is formulated as:

1. All observations $\left\{X_{i}\right\}$ are independent.

2. Each observation is normally distributed, $X_{i} \sim \mathcal{N}\left(\mu_{i}, \sigma_{i}^{2}\right)$.

3. Mean $\mu_{i}$ and variance $\sigma_{i}^{2}$ are known.

We assume that at least one success, $X_{i}>\mu_{i}$ for some $i \in\{1,2, \ldots N\}$, has been observed. The set of observations $D=\left\{X_{i}\right\}$ is partitioned into subsets containing the success and failure runs, keeping only the former and ignoring the latter. Let $A_{j}$ denote the subset of the observations of the $j^{\text {th }}$ success run, $A_{j}=\left\{X_{j_{1}}, X_{j_{2}} \ldots\right\}$. The weight of the $j^{\text {th }}$ success run is then taken to be

$$
w\left(A_{j}\right) \equiv \chi_{\text {run }, j}^{2}=\sum_{i} \frac{\left(X_{i}-\mu_{i}\right)^{2}}{\sigma_{i}^{2}},
$$

where the sum over $i$ is understood to cover all $X_{i} \in A_{j}$. The test statistic is the largest weight of any success run

$$
T \equiv \max _{j} \chi_{\text {run }, j}^{2}
$$


Our goal is to calculate the $p$-value $p \equiv P\left(T \geq T_{\text {obs }} \mid N\right)=1-P\left(T<T_{\text {obs }} \mid N\right)$. Due to the symmetry of the normal distribution, for each observation the chance of success is

$$
P\left(X_{i} \text { is a success } \mid \mathcal{H}\right)=P\left(X_{i}>\mu_{i} \mid \mathcal{H}\right)=\frac{1}{2} .
$$

The following analysis up to (16) is valid for any $\mathcal{H}$ such that (6) holds. This symmetric Bernoulli property drastically simplifies the calculation.

The key idea is that the set of all sequences of successes and failures in $N$ Bernoulli trials can be decomposed into equivalence classes, and $P(T<$ $\left.T_{\text {obs }} \mid N\right)$ can be expressed as an expectation value over inequivalent sequences.

For our purposes a sequence $\xi$ of length $N$ is sufficiently characterized by the numbers $n_{1}, \ldots n_{N}$ denoting the number of success runs of length one, $n_{1}$, of length two, $n_{2} \ldots$; we write $\mathbf{n}(\xi)=\left(n_{1}, \ldots, n_{N}\right)$. Two sequences $\xi_{1}, \xi_{2}$ of length $N$ are declared equivalent, if they have the same success runs; i.e.

$$
\xi_{1} \sim \xi_{2} \Leftrightarrow \mathbf{n}\left(\xi_{1}\right)=\left(n_{1}, \ldots, n_{N}\right)=\mathbf{n}\left(\xi_{2}\right)
$$

If the last $n_{N-k}, \ldots, n_{N}$ are zero they may be omitted. Reflexivity, symmetry and transitivity of $\sim$ follow immediately. To illustrate definition (7), consider the following example.

Let $\mathrm{S}\left[\mathrm{F}\right.$ ] denote a success [failure], and consider the sequences $\xi_{1}=$ SSSFFSFS and $\xi_{2}=$ FSFSSSFS. Both sequences exhibit two success runs of length one, $n_{1}=2$, and one success run of length three, $n_{3}=1$. Hence $\mathbf{n}\left(\xi_{1}\right)=(2,0,1)=\mathbf{n}\left(\xi_{2}\right)$, and the sequences are equivalent, $\xi_{1} \sim \xi_{2}$.

In order to find all inequivalent sequences that need to be accounted for it turns out to be most useful to fix the number of successes, $r$, and the number of success runs, $M$, with joint density $P(M, r \mid N)$. Thus by the law of total probability

$$
P\left(T<T_{o b s} \mid N\right)=\sum_{r=1}^{N} \sum_{M=1}^{M_{\max }} P\left(T<T_{o b s} \mid M, r, N\right) \cdot P(M, r \mid N) .
$$

The maximum number of success runs, $M_{\max }$, for fixed $r$ is determined as follows: there can be no more success runs than successes, so $M \leq r$. On the other hand, the success runs have to be separated by at least one failure, hence $M \leq N-r+1$. For a fixed number of observations, $N$, we have $M \leq\left\lfloor\frac{N+1}{2}\right\rfloor$. It is easily verified that the latter condition is implied by the 
first two, and the constraints are summarized as

$$
M_{\max }=\min (r, N-r+1) .
$$

The joint distribution $P(M, r \mid N)$ is conveniently expressed as

$$
P(M, r \mid N)=\frac{1}{2^{N}-1} \cdot R(M, r \mid N)
$$

where $R(M, r \mid N)$ denotes the number of (possibly equivalent) sequences with $M$ success runs and $r$ successes in $N$ Bernoulli trials. As an example consider $R(1,2 \mid 3)=|\{\mathrm{SSF}, \mathrm{FSS}\}|=2$. In fact $R(M, r \mid N)$ can be calculated efficiently by a recursive algorithm, but it will be seen to cancel out so that we have no need to compute it.

With $M, r, N$ fixed, we can decompose $P\left(T<T_{\text {obs }} \mid M, r, N\right)$ into the desired average over inequivalent sequences

$$
P\left(T<T_{o b s} \mid M, r, N\right)=\sum_{\pi} P\left(T<T_{o b s} \mid \pi\right) P(\pi \mid M, r, N) .
$$

The key observation is that the set of inequivalent sequences $\{\pi\} \subset\{\xi\}$ is in one-to-one correspondence with the set of integer partitions of $r$ into exactly $M$ summands.

Due to their widespread applicability, the integer partitions have been studied extensively: [15] devoted an entire book to the partitions. For an online overview we refer to [16]. Efficient algorithms to construct all partitions $\{\pi\}$ explicitly are well known; e.g. [17, 18]. These algorithms scale linearly with the number of partitions. We refer to the appendix for more details on integer partitions; there we derive the exact number of sequences needed in calculating $P\left(T<T_{\text {obs }} \mid N\right)$. It grows asymptotically as $\mathcal{O}\left(\frac{1}{N} e^{\sqrt{N}}\right)$.

The probability of one such sequence $\pi, P(\pi \mid M, r, N)$ is just its multiplicity, $W(\pi)$, divided by the total number of elements in $\{\xi\}$, which is $R(M, r \mid N)$. The multiplicity is found by basic urn model considerations as the product of the number of ways to shuffle the success runs and the number of ways to distribute the failures in between and around the success runs. While the former is just the multinomial coefficient

$$
\left(\begin{array}{c}
M \\
n_{1}, \ldots, n_{N}
\end{array}\right),
$$


the latter is obtained as a binomial coefficient. Given $M$ success runs and $N-r$ failures, $M-1$ failures are needed to separate the success runs, and the remaining $N-r-M+1$ failures can be allocated freely into the $M+1$ slots surrounding the success runs. Using Eq. 1 from [19] we obtain

$$
\begin{aligned}
W(\pi) & =\left(\begin{array}{c}
M \\
n_{1}, \ldots, n_{N}
\end{array}\right) \cdot\left(\begin{array}{c}
N-r+1 \\
M
\end{array}\right)=\frac{(N-r+1) !}{(N-r+1-M) ! \cdot \prod_{l} n_{l} !} \\
& =\frac{(N-r+2-M)_{M}}{\prod_{l} n_{l} !}
\end{aligned}
$$

with the Pochhammer symbol defined for positive integers $x, n$ as

$$
(x)_{n} \equiv \Gamma(x+n) / \Gamma(x)=(x+n-1) ! /(x-1) !
$$

Using the independence of the observations, the probability to observe a value of $T$ smaller than a fixed $T_{o b s}$ in an entire sequence is just the product of probabilities of finding a weight $w_{l}<T_{o b s}$ in each individual success run of length $l$, hence we find at once

$$
P\left(T<T_{o b s} \mid \pi\right)=\prod_{l}\left[P\left(w_{l}<T_{o b s} \mid l\right)\right]^{n_{l}} .
$$

As an example, consider again the sequence SSSFFSFS, with success runs distribution $\mathbf{n}=(2,0,1)$, then its contribution reads

$$
P\left(T<T_{o b s} \mid \pi\right)=P\left(w_{l}<T_{o b s} \mid l=1\right)^{2} P\left(w_{l}<T_{o b s} \mid l=3\right) .
$$

As an intermediate result we note

$$
\begin{aligned}
P\left(T<T_{o b s} \mid N\right) & =\sum_{r=1}^{N} \sum_{M=1}^{M_{\max }} \sum_{\pi} P\left(T<T_{o b s} \mid \pi\right) \cdot P(\pi \mid M, r, N) \cdot P(M, r \mid N) \\
& =\sum_{r=1}^{N} \sum_{M=1}^{M_{\max }} \sum_{\pi} \prod_{l}\left[P\left(w_{l}<T_{o b s} \mid l\right)\right]^{n_{l}} \cdot \frac{(N-r+2-M)_{M}}{\left(2^{N}-1\right) \cdot \prod_{l} n_{l} !} \\
M_{\max } & =\min (r, N-r+1)
\end{aligned}
$$

Eq. (16) is useful for generalizations where $P\left(X_{i}\right.$ is a success $\left.\mid \mathcal{H}\right)=\frac{1}{2}$ but the individual $X_{i}$ are not normally distributed, since at this point it is still 
left open which weight $w_{l}$ to use in order to quantify the discrepancy between the model prediction and the observed outcome of individual success runs.

Assuming $X_{i} \sim \mathcal{N}\left(\mu_{i}, \sigma_{i}^{2}\right)$, it is most natural to use the $\chi^{2}$ of each run because it corresponds directly to the probability density of the data. The additional benefit of this choice is that $P\left(T<T_{o b s} \mid l\right)$ is known exactly, it is just the cumulative distribution function of the celebrated $\chi^{2}$ - distribution with $l$ degrees of freedom:

$$
\begin{aligned}
& P\left(T<T_{o b s} \mid l\right)=\int_{0}^{T_{o b s}} \mathrm{~d} \chi^{2} \frac{1}{2^{l / 2} \Gamma(l / 2)} e^{-\chi^{2} / 2}\left(\chi^{2}\right)^{-1+l / 2} \\
& P\left(T<T_{o b s} \mid l\right)=\frac{\gamma\left(l / 2, T_{o b s} / 2\right)}{\Gamma(l / 2)} .
\end{aligned}
$$

In other words, it is the regularized incomplete gamma function, comprised of the lower incomplete gamma function

$$
\gamma(a, x)=\int_{0}^{x} \mathrm{~d} t t^{a-1} e^{-t}
$$

and the complete gamma function

$$
\Gamma(a)=\int_{0}^{\infty} \mathrm{d} t t^{a-1} e^{-t} .
$$

This is true even though the individual observations in a run are not normally distributed, but according to the half-normal distribution, since they are required to be successes. In fact, if $X_{i}$ is a random variable distributed according to a standard normal distribution limited to the domain $\left[a_{i}, b_{i}\right], a_{i}, b_{i} \in$ $\overline{\mathbb{R}}$, the sampling distribution of

$$
X_{1}^{2}+\cdots+X_{l}^{2}
$$

is given by the $\chi^{2}$ - distribution with $l$ degrees of freedom (17), regardless of the domains $\left[a_{i}, b_{i}\right]$. The proof follows the traditional lines by transforming to spherical coordinates. It is then seen that the angular contributions (depending on $a_{i}, b_{i}$ ) are removed in the normalization, and the radial behavior (independent of $a_{i}, b_{i}$ ) is the $\chi^{2}$ - distribution. See, e.g., [20, chap. 11] for details.

Now the derivation of the distribution of $T$ is completed, (16) combined with (17) give a complete specification that can be implemented in just a few 
lines of code in mathematica [21]. As an example, $P\left(T \geq T_{\text {obs }} \mid N=25\right)$ is plotted as a function of $T_{o b s}$ in Fig. 1. Since the number of partitions which contribute to $P\left(T<T_{o b s} \mid N\right)$ grows rapidly with $N$ (see appendix for details), we have to resort to a Monte Carlo approximation of the $p$-value for $N \gtrsim 80$. Note that the Monte Carlo output also serves as a valuable cross check with the exact solution for small $N$. We now briefly describe the Monte Carlo algorithm:

1. Fix a number of experiments, $K$, and the number of observations, $N$, in each experiment.

2. Generate $K \cdot N$ standard normal variates.

3. In each of the $K$ experiments, find the largest $\chi_{\text {run }}^{2}$ of any success run. This is $T_{o b s, j}$ for the experiment $j, j=1 \ldots K$. Filter out all experiments that contain no success.

4. Let $L$ denote the number of experiments in which $T_{o b s, j} \geq T_{o b s}$. Then estimate the $p$-value, $P\left(T \geq T_{\text {obs }} \mid N\right)$ as $p \approx \frac{L}{K}$.

We estimate the uncertainty on $p$ as obtained in step 4 from a Bayesian point of view. The sampling can be seen as a Bernoulli process, with a constant chance of $p$ in each trial $j$ that $T_{o b s, j} \geq T_{o b s}$. Assuming a uniform prior on $p$, the posterior then becomes

$$
P(p \mid L, K)=\frac{(K+1) !}{L !(K-L) !} p^{L}(1-p)^{K-L}
$$

with the mode at $p=L / K$. Let $\langle\cdot\rangle$ denote the expectation value under the posterior, then the variance of $p$ is

$$
\frac{\langle p\rangle(1-\langle p\rangle)}{K+3}
$$

Thus for large $K$, the variance falls off as $1 / K$.

As discussed in the introduction, we can define another statistic, call it $T^{f}$, analogous to $T$, but now for the failure runs instead of the success runs. $T^{f}$ also tests the model's ability to reproduce the data. In the algorithm indicated above, the same variates obtained in step 2 can be used to calculate $p$-values for $T^{f}$. One simply considers the largest $\chi_{\text {run }}^{2}$ of any failure run and filters out all experiments with no failure. Due to the symmetry of the Normal distribution, we have

$$
P\left(T<T_{o b s} \mid N\right)=P\left(T^{f}<T_{o b s} \mid N\right)
$$




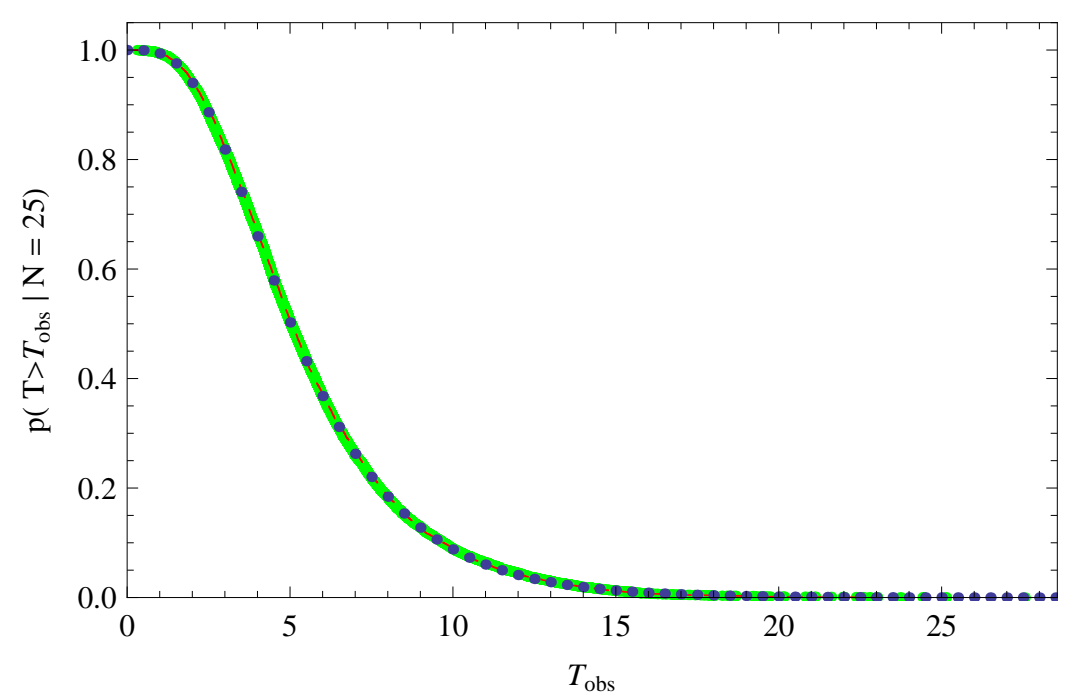

Figure 1: $p$-value for the runs test statistic $T$ and $N=25$ observations. The Monte Carlo results for successes (green) and failures (red dashed) with $K=10000$ generated experiments are in excellent agreement with the exact results (blue dotted) using (16), (17).

Given the set of samples $\left\{T_{o b s, j}\right\}$, we can construct the empirical cumulative distribution function (ECDF) [22, chap. 25.3] for graphical display. In Fig. 1, we show the Monte Carlo results $\left(1-\operatorname{ECDF}\left(T_{o b s}\right), K=10000, N=\right.$ 25) for success runs (green), and failure runs (red) and finally the exact results (blue) for $N=25$ data points.

For practical use, the critical values of $T$ for three often used confidence levels $\alpha=5 \%, 1 \%, 0.1 \%$ are presented in Table 2 . Note that for fixed $\alpha$, the critical values vary approximately linearly with $\log N$

$$
T_{\text {crit }}(N \mid \alpha) \sim c \cdot \log N+b(\alpha) .
$$

The slope $c$ appears to be nearly independent of $\alpha$. In Fig. 2, the following parameter values are chosen:

$$
\begin{gathered}
\alpha=0.05 \Rightarrow c=2.8, \quad b=2.5 \\
\alpha=0.01 \Rightarrow c=2.9, \quad b=6.1 \\
\alpha=0.001 \Rightarrow c=3.0, \quad b=11.6
\end{gathered}
$$




\begin{tabular}{c|ccccccc}
\hline $\mathbf{N}$ & 5 & 10 & 25 & 50 & 100 & 500 & 1000 \\
\hline $\boldsymbol{\alpha}=\mathbf{0 . 0 5}$ & 6.8 & 8.8 & 11.5 & 13.4 & 15.3 & 19.8 & 21.6 \\
$\boldsymbol{\alpha}=\mathbf{0 . 0 1}$ & 10.4 & 12.8 & 15.7 & 17.7 & 19.7 & 24.4 & 25.9 \\
$\boldsymbol{\alpha}=\mathbf{0 . 0 0 1}$ & 15.5 & 18.3 & 21.6 & 23.8 & 25.6 & 29.9 & 32.0 \\
\hline
\end{tabular}

Table 1: Critical values of $T_{o b s}$ at the $\alpha=5 \%, 1 \%, 0.1 \%$ level as a function of $N$. Up to $N=50$ these are found from the exact solution. For larger $N$, the critical values are estimated from the Monte Carlo approximation using $K=10^{5}$ simulated experiments and linear interpolation of $p\left(T_{o b s}\right)$ based on the points $\left(T_{o b s, j}, p\left(T_{o b s, j}\right)\right), j=1 \ldots K$.

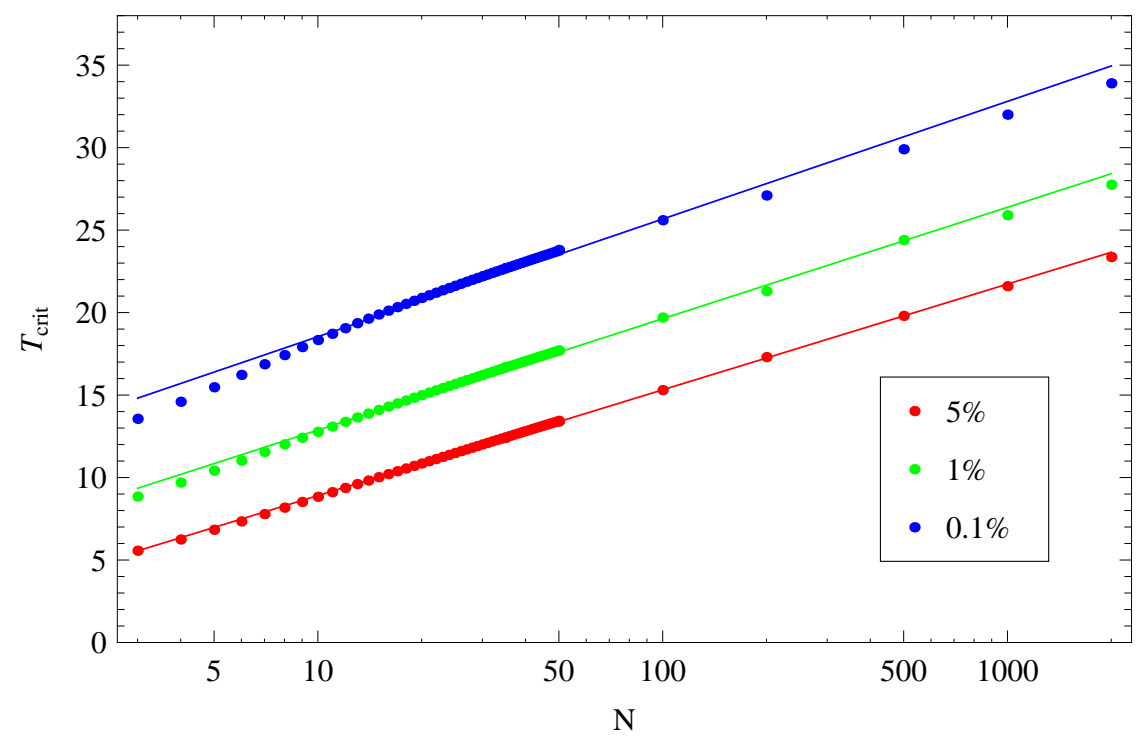

Figure 2: Critical values of $T_{o b s}$ at the $\alpha=5 \%, 1 \%, 0.1 \%$ level. $T_{\text {crit }}$ scales approximately linearly with $\log N$. The slope is nearly independent of $\alpha$. 


\section{Example}

Let us discuss an example that frequently arises in high energy physics to study the power of significance tests based on $T$. For comparison, we use the classic $\chi^{2}$ test statistic.

Assume an experiment is conducted to observe the quantity $y=y(x)$. The uncertainties are modeled as arising from a normal distribution with known variance, then for each of the $N$ independent observations

$$
y_{i} \sim \mathcal{N}\left(\mu_{i}, \sigma_{i}^{2}\right)
$$

The purpose of the experiment is to decide whether the currently accepted hypothesis $\mathcal{H}$ is sufficient to explain the data. The predictions derived from $\mathcal{H}$ are given as

$$
\mu_{i}=f\left(x_{i}\right)
$$

In addition, assume there exists an extension to $\mathcal{H}$, denoted by $\mathcal{H}_{1}$, whose predictions are

$$
\mu_{i}=f\left(x_{i}\right)+g\left(x_{i}\right) .
$$

Typically the extra contribution $g(x)$ is significant in a narrow region only. For concreteness, we assume it is a localized peak of the Cauchy-Lorentz form with location parameter $\beta$ and scale parameter $\gamma$

$$
g(x)=A \cdot\left(1+\frac{(x-\beta)^{2}}{\gamma^{2}}\right)^{-1} .
$$

The magnitude of the extra contribution is defined by $A$. Three cases are to be distinguished. For $A \rightarrow 0, \mathcal{H}_{1}=\mathcal{H}$. For fixed confidence level $\alpha$, tests based on $T$ and $\chi^{2}$ reject $\mathcal{H}$ with the nominal probability $\alpha$.

For $A \rightarrow \infty, \mathcal{H}$ is rejected with probability 1 for either statistic. In the most interesting region, $A$ not too small and not too large, we study the rejection power of $T$ and $\chi^{2}$ by simulating experiments under $\mathcal{H}_{1}$. We then analyze the data under $\mathcal{H}$ and estimate the power as the fraction of times the $p$-value is found in the rejection region defined by the confidence level $\alpha=0.05$. We simulate an ensemble of 10000 experiments with $N=10$ draws from $\mathcal{H}_{1}$ with $x_{i}=i, i=1 \ldots 10$ and parameters $\beta=5.5, \gamma=2$ fixed for different values of $A$. Without loss of generality, we choose $f(x)=0, \sigma_{i}=1$. The numerical results are shown in Figure 3 as a function of $A$. The power of $T$ equals the power of $\chi^{2}$ for $A=0$ and $A \gg 1$ as expected. In the 


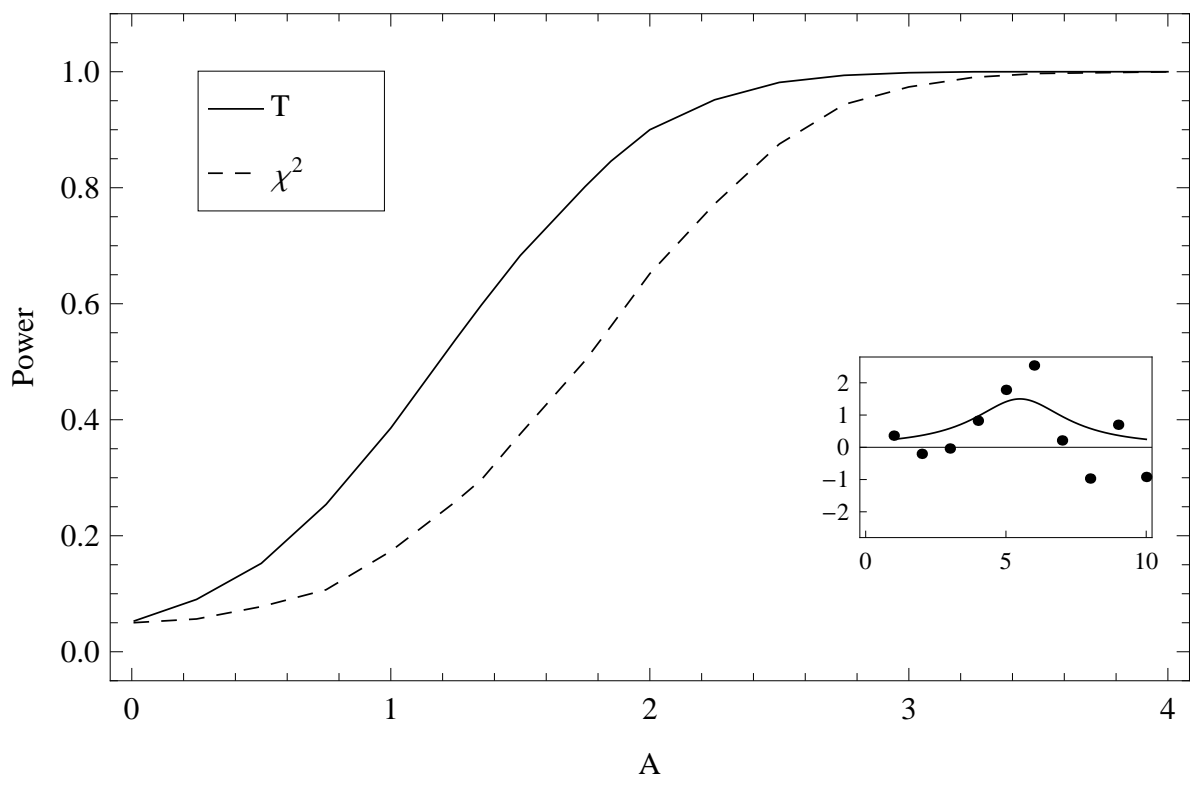

Figure 3: Power of statistics $T$ and $\chi^{2}$ in rejecting the null hypothesis of normality around zero mean at the $5 \%$ confidence level. For fixed $A, 10000$ experiments, each of sample size 10, have been generated from a normal distribution with variance one. The mean of sample $i, i=1 \ldots 10$ is distributed according to a Cauchy distribution $A \cdot\left(1+\frac{(i-5.5)^{2}}{3^{2}}\right)^{-1}$. A sample data set $(A=1.5)$ is shown in the inset. The curves show the power as a function of the amplitude $A$.

intermediate region, the power of $T$ significantly exceeds that of $\chi^{2}$. Similar results are obtained when keeping $A$ fixed and varying $\gamma$ instead.

Moreover, if we choose a distribution with light tails (e.g. a normal distribution) for $g(x)$ instead of the heavy-tailed Cauchy distribution, the qualitative results are unaffected. The power of $T$ is larger than the power of $\chi^{2}$ for the alternative $\mathcal{H}_{1}$. For medium sized $g(x)$, the difference can reach up to $40 \%$.

\section{Discussion}

We have introduced the test statistic $T$ and calculated its distribution for the case of a sequence of independent observations, each following a normal distribution with known mean and variance. Implementing the algorithm to calculate critical values of $T$ for the various confidence levels is straightforward, but the execution time grows rapidly with the number of observations 
$N$. Hence a Monte Carlo scheme is recommended to calculate critical values for $N \gtrsim 80$, yielding results in reasonable time even for $N \gtrsim 1000$, thus covering virtually the whole range of interest relevant to everyday problems. We have verified that the Monte Carlo results agree well with exact results for small $N$.

We have demonstrated the usefulness of $T$ and recommend its usage for hypothesis testing especially against alternatives with additional local peaks.

The more common problem in data analysis is to consider a composite hypothesis: in a first step free parameters of the model are estimated from the data ("fit") and in the second step predictions, based on the fitted parameters, and observations are compared ("goodness of fit"). With most test statistics the effect of fitted parameters on the sampling distribution of the statistic is not analytically known. The only notable exception to this rule is the $\chi^{2}$ statistic: for $k$ parameters extracted from maximizing the likelihood of $N$ normal observations, the number of degrees of freedom is $N-k$, instead of $N$ in case all parameters are known a priori. Unfortunately, this cannot be extended to the runs statistic $T$ considered here. However what we can do is to simulate data sets using a Monte Carlo approach, and study the approximate numerical distribution of $T$. For the simplest case of a straight line and a maximum likelihood fit to 10 data points, the results are shown in Fig. 4. It is evident that $p(T)$ drops to zero much more sharply for the fitted data (green=successes, red=failures) than for the exact results with no parameters fitted (blue). Accordingly, the critical values for fitted $T$ at level $\alpha=5 \%, 1 \%, 0.1 \%$ are $T_{\text {crit }}=6.0,8.5,12.4$. In general, the qualitative effect of fitting parameters but pretending that they were known before the data was taken is that the $p$-value is not distributed uniformly. Instead, its distribution is biased towards $p=1$, leading to conservative decisions. The quantitative effect depends on the number of observations and parameters, the maximization condition determining the best fit parameters (likelihood, posterior ...) and possibly other effects.

Through Monte Carlo approximations the use of the runs statistic $T$ can be further generalized to the important class of problems involving asymmetric uncertainties like Binomial or Poisson distributions. All that needs to be changed is the weight of individual runs. As a starting point one could define $T$ as the smallest probability (density) of any run, $T=\min _{j} P\left(A_{j} \mid \mathcal{H}\right)$. Numerically the distribution of $T$ is then found in analogous fashion to the algorithm described in the caption of Fig. 4. An implementation of this algorithm is scheduled to be included in a future release of BAT, the Bayesian 


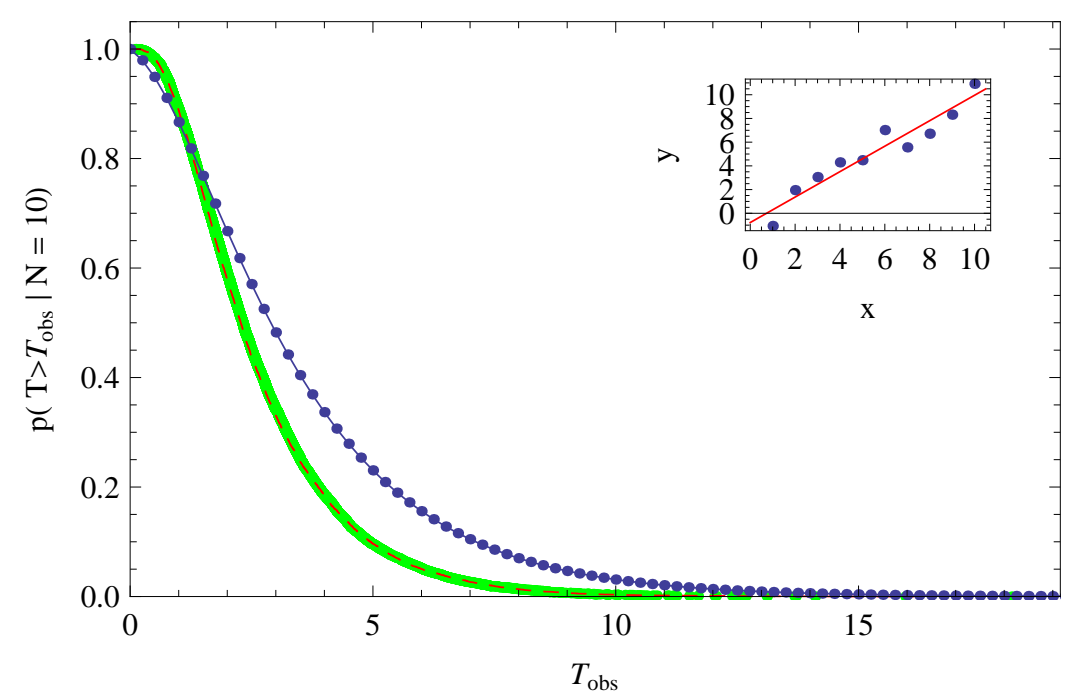

Figure 4: Distribution of runs test statistic $T$ with and without fitted parameters. The Monte Carlo results for successes (green) and failures (red dashed) are obtained from $K=10000$ generated experiments. Each data set consists of $N=10$ data points $\left(x_{i}, y_{i}\right)$, where the $y_{i}$ are normally distributed around a straight line of unit slope and zero intercept, $y_{i} \sim \mathcal{N}\left(\mu=1 \cdot x_{i}+0, \sigma^{2}=1\right)$. Then a maximum likelihood fit is performed to extract the two parameters of a straight line model $y=m \cdot x+b$ (see inset). Finally the predictions are calculated from the fitted model, and $T_{\text {obs }}$ is determined for each experiment. With the set of 10000 values of $T_{o b s}$ the empirical CDF (ECDF) is computed, and $1-\operatorname{ECDF}\left(T_{o b s}\right)$ is plotted. For comparison the exact results (blue dotted) for $N=10$ using Eq. (16), (17) are shown. The effect of fitting is that $p(T)$ drops more sharply, hence the critical values are pushed towards smaller $T$; e.g. at the $5 \%$ level $T_{\text {crit }}=6.0$ (fit) vs $T_{\text {crit }}=8.8$ (no fit).

Analysis Toolkit [23]. BAT is a $\mathrm{C}++$ library based on the Markov Chain Monte Carlo approach which offers routines for fitting, limit setting, goodness of fit and more. Using the Metropolis algorithm [24] it is possible to simulate the data sets needed for approximate $p$-value calculations.

\section{Appendix}

\section{Integer Partitions and Computational Complexity}

We are now interested in the number of sequences, $\nu(N)$, which need to be taken into account to calculate a $p$-value for $T, P\left(T \geq T_{o b s} \mid N\right)$, using 
(16). Put differently, $\nu(N)$ is the number of terms in the multiple sum

$$
P\left(T \geq T_{o b s} \mid N\right)=1-\sum_{r=1}^{N} \sum_{M=1}^{\min (r, N-r+1)} \sum_{\pi} \ldots
$$

where $\sum_{\pi}$ extends over all inequivalent sequences with $r$ successes distributed in $M$ success runs, see (7) and (11). Since $\nu(N)$ determines the number of steps needed to calculate the $p$-value on a computer, knowing the form of the $N$-dependence aids in ascertaining whether the computer can be expected to finish the calculation in reasonable time. In the main result of this section, Proposition 1, $\nu(N)$ is essentially given by the number of integer partitions. To begin with, we introduce the integer partitions and illustrate with an example. The book [15] by Andrews is a good reference devoted entirely to partitions.

Definition 1. Let Part $(\mathrm{N})$ denote the number of partitions of the integer $N$ into a sum of one or more positive integers. For consistency it is useful to define Part $(0) \equiv 1$. Let Part $(\mathrm{N}, \mathrm{k})$ denote the number of partitions of $N$ into exactly $k$ addends and finally let $\operatorname{Part}_{\leq}(\mathrm{N}, \mathrm{i})$ denote the number of partitions of $N$ into integers of at most size $i$, with $\operatorname{Part}_{\leq}(0, \mathrm{i}) \equiv 1$.

Example 1. The integer 5 can be written in Part $(5)=7$ different ways:

$$
\begin{aligned}
5 & =5 \\
& =4+1 \\
& =3+2 \\
& =3+1+1 \\
& =2+2+1 \\
& =2+1+1+1 \\
& =1+1+1+1+1
\end{aligned}
$$

One can see that 5 can be decomposed as a sum of exactly three non-zero integers in two ways (Eq. (A.5) and (A.6)), thus Part $(5,3)=2$. Furthermore, the number of ways to partition 5 into addends less than 3 is Part $_{\leq}(5,2)=3$ (Eq. (A.6)- (A.8)).

The three partition numbers just defined are obviously closely connected, we shall need the following relations; elementary proofs based on Ferrer's 
diagrams can be found in the books by Andrews [15, chap. 1] and Knuth [17, chap. 7.2.1.4].

Fact 1. Assuming $N \geq 1$, Def. 1 yields:

$$
\begin{gathered}
\operatorname{Part}(\mathrm{N})=\sum_{r=1}^{N} \operatorname{Part}(\mathrm{N}, \mathrm{r}) \\
\operatorname{Part}_{\leq}(\mathrm{N}, \mathrm{r})=\sum_{M=1}^{r} \operatorname{Part}(\mathrm{N}, \mathrm{M}) \\
\operatorname{Part}_{\leq}(\mathrm{M}, \mathrm{r}-\mathrm{M})=\operatorname{Part}(\mathrm{r}, \mathrm{r}-\mathrm{M}) \\
\operatorname{Part}_{(\mathrm{N})}=\sum_{r=0}^{N-1} \operatorname{Part}_{\leq}(\mathrm{r}, \mathrm{N}-\mathrm{r})
\end{gathered}
$$

Proposition 1. Let $\nu(N)$ denote the number of inequivalent Bernoulli sequences of length $N$, where the probability of a success is $\frac{1}{2}$ in each trial and the equivalence relation is defined in (17). Then

$$
\begin{aligned}
\nu(N) & \equiv \sum_{r=1}^{N} \sum_{M=1}^{\min (r, N-r+1)} \operatorname{Part}(\mathrm{r}, \mathrm{M}) \\
& =\operatorname{Part}(\mathrm{N}+1)-1
\end{aligned}
$$

Proof. We start from the right hand side of the proposition using (A.12):

$$
\begin{aligned}
\operatorname{Part}(\mathrm{N}+1)-1 & =-1+\sum_{r=0}^{N} \operatorname{Part}_{\leq}(\mathrm{r}, \mathrm{N}+1-\mathrm{r}) \\
& =\sum_{r=1}^{N} \operatorname{Part}_{\leq}(\mathrm{r}, \mathrm{N}+1-\mathrm{r}) .
\end{aligned}
$$

Now using (A.10):

$$
\operatorname{Part}(\mathrm{N}+1)-1=\sum_{r=1}^{N} \sum_{M=1}^{N-r+1} \operatorname{Part}(\mathrm{r}, \mathrm{M}) .
$$

But we know that we cannot partition $r$ successes into more than $r$ success runs, so Part $(r, M>r)=0$, hence 


$$
\begin{aligned}
\operatorname{Part}(\mathrm{N}+1)-1 & =\sum_{r=1}^{N} \sum_{M=1}^{\min (r, N-r+1)} \operatorname{Part}(\mathrm{r}, \mathrm{M}) \\
& =\nu(N) .
\end{aligned}
$$

Since Part (r, M) represents the number of elements in $\sum_{\pi}$ of (A.1), $\nu(N)$ is the exact number of sequences that contribute to $P\left(T \geq T_{o b s} \mid N\right)$. We can approximate $\nu(N)$ by employing the asymptotic expression of Part $(\mathrm{N})$ for large $N$ first derived by Hardy and Ramanujan [25]:

$$
\operatorname{Part}(\mathrm{N}) \sim \frac{\exp (\pi \sqrt{2 / 3 \cdot N})}{4 \sqrt{3} N} .
$$

Hence, for large $N, \nu(N)$ grows nearly exponentially.

Corollary 1. For large $N, \nu(N)$ is approximately given by

$$
\nu(N) \sim \frac{\exp (\pi \sqrt{2 / 3 \cdot(N+1)})}{4 \sqrt{3}(N+1)}
$$

This implies that for large $N$ (say $N=1000$ ), in equations (8), (11) the sum is over more partitions $\left(\nu(N)=2.5 \times 10^{31} \approx 2^{104}\right)$ than a current 64 bit desktop computer could even address in memory. In practice the exact evaluation of $P\left(T \geq T_{\text {obs }} \mid N\right)$ becomes too slow already for $N \gtrsim 80$ where $\nu(80)=1.8 \times 10^{7}$. In contrast a Monte Carlo solution based on sampling a large number of batches, $K$, each with $N$ pseudo random numbers is much faster: its computational complexity is $\mathcal{O}(K \cdot N)$.

\section{References}

[1] A. M. Mood, The distribution theory of runs, The Annals of Mathematical Statistics 11 (4) (1940) 367-392.

[2] N. Balakrishnan, M. V. Koutras, Runs and scans with applications, J. Wiley, 2002. 
[3] M. V. Koutras, Applications of Markov chains to the distribution theory of runs and patterns, Vol. 21 of Handbook of Statistics, Elsevier Science, Amsterdam, 2002, pp. 431-472.

[4] J. C. Fu, W. Y. W. Lou, Distribution theory of runs and patterns and its applications, World Scientific, 2003.

[5] E. J. Burr, G. Cane, Longest run of consecutive observations having a specified attribute, Biometrika 48 (3/4) (1961) 461-465.

[6] A. N. Philippou, F. S. Makri, Successes, runs and longest runs, Statistics \& Probability Letters 4 (2) (1986) 101-105.

[7] M. Muselli, Simple expressions for success run distributions in bernoulli trials, Statistics \& Probability Letters 31 (2) (1996) 121-128.

[8] J. C. Fu, M. V. Koutras, Distribution theory of runs: A markov chain approach, Journal of the American Statistical Association 89 (427) (1994) 1050-1058.

[9] W. Y. W. Lou, On runs and longest run tests: A method of finite markov chain imbedding, Journal of the American Statistical Association 91 (436) (1996) 1595-1601.

[10] E. Vaggelatou, On the length of the longest run in a multi-state markov chain, Statistics \& Probability Letters 62 (3) (2003) 211-221.

[11] J. C. Fu, L. Wang, W. Y. W. Lou, On exact and large deviation approximation for the distribution of the longest run in a sequence of Two-State markov dependent trials, Journal of Applied Probability 40 (2) (2003) 346-360.

[12] S. Eryilmaz, Some results associated with the longest run statistic in a sequence of markov dependent trials, Applied Mathematics and Computation 175 (1) (2006) 119-130.

[13] S. Eryilmaz, S. Demir, Success runs in a sequence of exchangeable binary trials, Journal of Statistical Planning and Inference 137 (9) (2007) 29542963. 
[14] F. S. Makri, A. N. Philippou, Z. M. Psillakis, Success run statistics defined on an urn model, Advances in Applied Probability 39 (4) (2007) 991-1019.

[15] G. E. Andrews, The theory of partitions, Cambridge University Press, 1998.

[16] N. J. A. Sloane, The On-Line encyclopedia of integer sequences, published electronically at http://www.research.att.com/ ${ }^{n}$ njas/sequences/A008284 (2010).

[17] D. E. Knuth, The Art of Computer Programming, Volume 4, Fascicle 3: Generating All Combinations and Partitions, Addison-Wesley Professional, 2005.

[18] M. A. Adnan, M. S. Rahman, et al., Distribution of distinguishable objects to bins: generating all distributions, International Journal of Computer Mathematics 84 (7) (2007) 953-965.

[19] F. S. Makri, A. N. Philippou, Z. M. Psillakis, Shortest and longest length of success runs in binary sequences, Journal of Statistical Planning and Inference 137 (7) (2007) 2226-2239.

[20] A. Stuart, J. K. Ord, Kendall's advanced theory of statistics. Vol. 1: Distribution theory, Oxford University Press, New York, 1994.

[21] Wolfram Research Inc., Mathematica Version 7.0, Champaign, Illinois, 2008.

[22] H. Cramer, Mathematical methods of statistics, Princeton Univ Pr, 1999.

[23] A. Caldwell, D. Kollár, K. Kröninger, BAT - the Bayesian analysis toolkit, Computer Physics Communications 180 (11) (2009) 2197-2209.

[24] N. Metropolis, A. W. Rosenbluth, A. H. Teller, E. Teller, M. N. Rosenbluth, J. Chem. Phys 21 (1953) 1087.

[25] G. H. Hardy, S. Ramanujan, Asymptotic formulae in combinatory analysis, Proceedings of the London Mathematical Society 2 (1) (1918) 75. 